\title{
A New Numerical Approximation Method for Two-Dimensional Wave Equation with Neumann Damped Boundary
}

\author{
Jiankang Liu $\mathbb{D}^{1,2}$ and Suying Zhang $\mathbb{D}^{1}$ \\ ${ }^{1}$ Institute of Theoretical Physics, Shanxi University, Taiyuan 030006, Shanxi, China \\ ${ }^{2}$ School of Mathematical Science, Shanxi University, Taiyuan 030006, Shanxi, China \\ Correspondence should be addressed to Suying Zhang; zhangsy@sxu.edu.cn
}

Received 27 October 2019; Revised 13 April 2020; Accepted 27 April 2020; Published 1 June 2020

Academic Editor: Honglei Xu

Copyright (C) 2020 Jiankang Liu and Suying Zhang. This is an open access article distributed under the Creative Commons Attribution License, which permits unrestricted use, distribution, and reproduction in any medium, provided the original work is properly cited.

In this paper, a fully discretized finite difference scheme is derived for two-dimensional wave equation with damped Neumann boundary condition. By discrete energy method, the proposed difference scheme is proven to be of second-order convergence and of unconditional stability with respect to both initial conditions and right-hand term in a proper discretized $L^{2}$ norm. The theoretical result is verified by a numerical experiment.

\section{Introduction}

In this paper, we consider the finite difference discretization for the following initial boundary value problem (IBVP) of wave equation in a square $\Omega=(0,1) \times(0,1)$ :

$$
w_{t t}(x, y, t)-\Delta w(x, y, t)=f(x, y, t), \quad(x, y, t) \in \Omega \times(0, T],
$$

$$
\begin{gathered}
w(x, y, 0)=\varphi(x, y), \\
w_{t}(x, y, 0)=\psi(x, y), \\
(x, y) \in \Omega, \\
w(0, y, t)=0, \\
w_{x}(1, y, t)=-w_{t}(1, y, t), \\
y \in[0,1], t \in[0, T], \\
w(x, 0, t)=0, \\
w_{y}(x, 1, t)=-w_{t}(x, 1, t), \\
x \in[0,1], t \in[0, T],
\end{gathered}
$$

where $\varphi(x, y), \psi(x, y)$, and $f(x, y, t)$ are given sufficient smooth functions, and $\varphi(0, y)=\varphi(x, 0)=0, \quad \psi(0, y)=$ $\psi(x, 0)=0, \quad \varphi_{x}(1, y)+\psi(1, y)=\varphi_{y}(x, 1)+\psi(x, 1)=0$, which means that the initial conditions are compatible with boundary conditions.

This system arises in many important models for distributed parameter control systems. In particular, in the model of a vibrating flexible membrane, the solution $w$ represents the transverse displacement of the membrane, and in models for acoustic pressure fields, the solution $w$ represents the fluid pressure (see, $[1,2]$ for more examples). The boundary conditions on the right and top sides of $\Omega$ in (3) and (4) are called Neumann actuations with damped controller from the viewpoint of control theory. Here, this kind of boundary condition is regarded as damped Neumann boundary. For this special boundary condition, it is more complicated to construct a proper energy function to prove a priori estimate of proposed finite difference scheme than that of classical boundary conditions (Dirichlet, Neumann, or Robin).

The numerical analysis of second-order hyperbolic equations has been extensively studied for decades. Many numerical methods have been used to approximate this kind of problem, including Finite Element Methods [3, 4], Finite Volume Methods [5, 6], Finite Difference Methods [7, 8], Discontinuous Galerkin methods [9-11], Mixed Finite Element Methods [12] and so on. In [4], wave equation with 
homogeneous boundary condition is considered, in which a posteriori error bounds in the $L^{\infty}\left(L^{2}\right)$ norm for finite element methods is derived under minimal regularity assumptions. A finite volume scheme on the nonconforming meshes for multidimensional wave equation is constructed in [6], where the error estimates for the approximations of the exact solution and its first derivatives is derived. An unconditionally stable and secondorder convergent finite difference scheme is constructed for one-dimensional linear hyperbolic equation in [8]. As far as hyperbolic conservation law is concerned, there are many distinguished papers focused on this topic, for example, the classical ENO/WENO schemes [13], the improved ENO/WENO schemes [14-17], and so on. All linear numerical schemes are either dispersive or dissipative. The computational dispersion can lead to noise in the numerical solution. Dispersion and dissipation phenomena were investigated in [18-22], where some composite schemes were proposed to reduce the dispersive effect on the numerical solution. High order compact finite difference scheme is an efficient way for solving PDEs. In [23-25], the high order compact finite difference scheme was constructed for hyperbolic equations subject to homogeneous boundary condition.

There are also some investigations of the numerical methods for viscous or strongly damped hyperbolic equation [26-29]. However, most of them just considered the homogeneous boundary problems. In [3], the generalized version of this kind of boundary condition in [3, (2.2b)]) was considered in the context of finite element methods. In [30], the mixed finite element formulation for second-order hyperbolic equation with absorbing boundary condition was investigated. Recently, the weak formulation of hyperbolic problems with inhomogeneous Dirichlet and Neumann boundary was considered in [31]. To the best of our knowledge, there is no contribution to the finite difference approximation to the $2 \mathrm{D}$ wave equation with damped Neumann boundary condition so far.

The rest of this paper is organized as follows. Some basic notations and lemmas are given in Section 2, which is essential to the analysis of finite difference schemes. Section 3 constructs the finite difference scheme for (1)-(4). A priori estimate of the proposed difference scheme is shown in Section 4. The unique solvability, convergence, and stability of proposed finite difference scheme are proved in Section 5. A numerical experiment is conducted in Section 6, before a conclusion is stated in Section 7.

\section{Preliminary}

Let $m$ and $n$ be two positive integers and assume that the space step and time step are $h=1 / m$ and $\tau=T / n$, respectively. Define $\Omega_{h}=\left\{\left(x_{i}, y_{j}\right) \mid x_{i}=i h, y_{j}=j h, 0 \leq i, j \leq m\right\}$, $\Omega_{\tau}=\left\{t_{k} \mid t_{k}=k \tau, 0 \leq k \leq n\right\} \quad$ and $\quad \Omega_{h \tau}=\Omega_{h} \times \Omega_{\tau}$. Let $w=\left\{w_{i, j}^{k} \mid 0 \leq i, j \leq m, 0 \leq k \leq n\right\}$ be the grid function on $\Omega_{h \tau}$. We introduce the following difference and averaging operators:

$$
\begin{aligned}
w_{i j}^{k+(1 / 2)} & =\frac{w_{i j}^{k}+w_{i j}^{k+1}}{2}, \\
\delta_{t} w_{i j}^{k+(1 / 2)} & =\frac{w_{i j}^{k+1}-w_{i j}^{k}}{\tau}, \\
D_{t} w_{i j}^{k} & =\frac{w_{i j}^{k+1}-w_{i j}^{k-1}}{2 \tau}, \\
\delta_{t}^{2} w_{i j}^{k} & =\frac{\delta_{t} w_{i j}^{k+(1 / 2)}-\delta_{t} w_{i j}^{k-(1 / 2)}}{\tau}, \\
\delta_{x} w_{i+(1 / 2), j}^{k} & =\frac{w_{i+1, j}^{k}-w_{i, j}^{k}}{h}, \\
\delta_{y} w_{i, j+(1 / 2)}^{k} & =\frac{w_{i, j+1}^{k}-w_{i, j}^{k}}{h}, \\
\delta_{x}^{2} w_{i j}^{k} & =\frac{\delta_{x} w_{i+(1 / 2), j}^{k}-\delta_{x} w_{i-(1 / 2), j}^{k}}{h}, \\
\delta_{y}^{2} w_{i j}^{k} & =\frac{\delta_{y} w_{i, j+(1 / 2)}^{k}-\delta_{y} w_{i, j-(1 / 2)}^{k}}{h}, \\
w_{i j}^{\bar{k}} & =\frac{w_{i j}^{k+1}+w_{i j}^{k-1}}{2} .
\end{aligned}
$$

The following lemmas (Lemma 1-Lemma 4) are necessary for analyzing the truncation error of the difference scheme and for proving the convergence of the difference scheme, which are analogous to lemmas in [32].

Lemma 1. Let $h>0$ and $c$ be two constants.

(a) If $g(x) \in C^{2}[c-h, c+h]$, then

$$
g(c)=\frac{1}{2}[g(c-h)+g(c+h)]-\frac{h^{2}}{2} g^{\prime \prime}\left(\xi_{0}\right), \quad c-h<\xi_{0}<c+h .
$$

(b) If $g(x) \in C^{2}[c, c+h]$, then

$$
g^{\prime}(c)=\frac{1}{h}[g(c+h)-g(c)]-\frac{h}{2} g^{\prime \prime}\left(\xi_{1}\right), \quad c-h<\xi_{1}<c+h .
$$

(c) If $g(x) \in C^{2}[c-h, c]$, then

$$
g^{\prime}(c)=\frac{1}{h}[g(c)-g(c-h)]+\frac{h}{2} g^{\prime \prime}\left(\xi_{2}\right), \quad c-h<\xi_{2}<c .
$$

(d) If $g(x) \in C^{3}[c-h, c+h]$, then

$$
g^{\prime}(c)=\frac{1}{2 h}[g(c+h)-g(c-h)]-\frac{h^{2}}{6} g^{\prime \prime \prime}\left(\xi_{3}\right), \quad c-h<\xi_{3}<c+h .
$$

(e) If $g(x) \in C^{4}[c-h, c+h]$, then 


$$
\begin{aligned}
g^{\prime \prime}(c)= & \frac{1}{h^{2}}[g(c+h)-2 g(c)+g(c-h)]-\frac{h^{2}}{12} g^{(4)}\left(\xi_{4}\right), \\
& c-h<\xi_{4}<c+h .
\end{aligned}
$$

(f) If $g(x) \in C^{3}[c-h, c]$, then

$$
\begin{aligned}
g^{\prime \prime}(c)= & \frac{2}{h}\left[g^{\prime}(c)-\frac{g(c)-g(c-h)}{h}\right]+\frac{h}{3} g^{\prime \prime \prime}\left(\xi_{5}\right), \\
& c-h<\xi_{5}<c .
\end{aligned}
$$

Lemma 2 (Gronwall Inequality). Suppose $\left\{F^{k}, G^{k} \mid k \geq 0\right\}$ are nonnegative sequences such that $F^{k+1} \leq(1+$ $c \tau) F^{k}+\tau G^{k}, k=0,1,2, \ldots$, then

$$
F^{k} \leq \exp (c k \tau)\left(F^{0}+\tau \sum_{l=1}^{k-1} G^{l}\right), \quad k=0,1,2, \ldots,
$$

where $c$ is a nonnegative constant.

Lemma 3. Suppose the mesh grids be $I_{h}=\left\{x_{i} \mid 0 \leq i \leq m\right\}$, where $x_{i}=i h, h=1 / m$. Let $u=\left\{u_{i} \mid 0 \leq i \leq m\right\}$ be mesh function on $I_{h}$ such that $u_{0}=0$, then

$$
\|u\|_{\infty} \leq|u|_{1}, \quad\|u\| \leq \frac{\sqrt{2}}{2}|u|_{1},
$$

where $\quad|u|_{1}=\sqrt{h \sum_{i=1}^{m}\left(\delta_{x} u_{i-(1 / 2)}\right)^{2}}, \quad\|u\|=$ $\sqrt{h\left((1 / 2) u_{0}^{2}+\sum_{i=1}^{m-1} u_{i}^{2}+(1 / 2) u_{m}^{2}\right)},\|u\|_{\infty}=\max _{0 \leq i \leq m}\left|u_{i}\right|$.

Proof. For $1 \leq i \leq m$, we have

$$
u_{i}=\sum_{j=1}^{i}\left(u_{j}-u_{j-1}\right)=h \sum_{j=1}^{i} \delta_{x} u_{j-(1 / 2)} .
$$

Square both sides of (14) and apply the Cauchy-Schwarz inequality, then

$$
\begin{aligned}
\left(u_{i}\right)^{2} & \leq\left(h \sum_{j=1}^{i} 1^{2}\right)\left(h \sum_{j=1}^{i}\left(\delta_{x} u_{j-(1 / 2)}\right)^{2}\right) \\
& =x_{i} h \sum_{j=1}^{i}\left(\delta_{x} u_{j-(1 / 2)}\right)^{2} \leq h \sum_{j=1}^{m}\left(\delta_{x} u_{j-(1 / 2)}\right)^{2},
\end{aligned}
$$

which directly implies

$$
\|u\|_{\infty} \leq|u|_{1} .
$$

The first inequality of (15) implies

$$
u_{i}^{2} \leq x_{i} h \sum_{j=1}^{i}\left(\delta_{x} u_{j-(1 / 2)}\right)^{2} \leq x_{i}|u|_{1}^{2} .
$$

Multiply both sides of (17) by $h$, and sum for $i$ from 1 to $m-1$, then

$$
h \sum_{i=1}^{m-1} u_{i}^{2} \leq h \sum_{i=1}^{m-1} x_{i}|u|_{1}^{2}=\frac{1}{2} m(m-1) h^{2}|u|_{1}^{2} .
$$

Thus, we have

$$
\|u\|^{2}=h \sum_{i=1}^{m-1} u_{i}^{2}+\frac{h}{2} u_{m}^{2} \leq\left(\frac{h}{2} m(m-1) h+\frac{h}{2}\right)|u|_{1}^{2}=\frac{1}{2}|u|_{1}^{2},
$$

which implies

$$
\|u\| \leq \frac{\sqrt{2}}{2}|u|_{1},
$$

and ends the proof.

Introduce a grid function space on $\Omega_{h}$ :

$$
\mathscr{V}=\left\{v \mid v=\left(v_{i j}\right)_{i, j=0}^{m} ; v_{i, 0}=0,0 \leq i \leq m ; v_{0, j}=0,1 \leq j \leq m\right\} .
$$

Then for $v \in \mathscr{V}$, define the norms

$$
\begin{gathered}
\|v\|=\sqrt{h^{2} \sum_{i, j=1}^{m-1}\left(v_{i j}\right)^{2}+h^{2} \frac{1}{2} \sum_{j=1}^{m-1}\left(v_{m j}\right)^{2}+h^{2} \frac{1}{2} \sum_{i=1}^{m-1}\left(v_{i m}\right)^{2}+h^{2} \frac{1}{4}\left(v_{m m}\right)^{2}}, \\
\left\|\delta_{x} v\right\|=\sqrt{h^{2} \sum_{i=0}^{m-1}\left(\frac{1}{2}\left(\delta_{x} v_{i+(1 / 2), 0}\right)^{2}+\sum_{j=1}^{m-1}\left(\delta_{x} v_{i+(1 / 2), j}\right)^{2}+\frac{1}{2}\left(\delta_{x} v_{i+(1 / 2), m}\right)^{2}\right)} \\
\left\|\delta_{y} v\right\|=\sqrt{h^{2} \sum_{j=0}^{m-1}\left(\frac{1}{2}\left(\delta_{y} v_{0, j+(1 / 2)}\right)^{2}+\sum_{i=1}^{m-1}\left(\delta_{y} v_{i, j+(1 / 2)}\right)^{2}+\frac{1}{2}\left(\delta_{y} v_{m, j+(1 / 2)}\right)^{2}\right)} .
\end{gathered}
$$


Lemma 4. Let $v=\left\{v_{i j} \mid 0 \leq i, j \leq m\right\}$ be mesh function on $\Omega_{h}$ such that $v_{0 j}=v_{i 0}=0$ for $1 \leq i, j \leq m$, then we have

$$
\|v\| \leq \frac{1}{2}|v|_{1}
$$

where $|v|_{1}=\left(\left\|\delta_{x} v\right\|^{2}+\left\|\delta_{y} v\right\|^{2}\right)^{1 / 2}$.

Proof. From Lemma 3, we have

$$
h \sum_{i=1}^{m-1} u_{i j}^{2}+\frac{h}{2} u_{m j}^{2} \leq \frac{1}{2} h \sum_{i=0}^{m-1}\left(\delta_{x} u_{i+(1 / 2), j}\right)^{2}, \quad 1 \leq j \leq m .
$$

Multiplying both sides of (24) by $h$ and summing for $j$ from 1 to $m-1$, we get

$$
h^{2} \sum_{i, j=1}^{m-1} u_{i j}^{2}+\frac{h^{2}}{2} \sum_{j=1}^{m-1} u_{m j}^{2} \leq \frac{1}{2} h^{2} \sum_{i=0}^{m-1} \sum_{j=1}^{m-1}\left(\delta_{x} u_{i+(1 / 2), j}\right)^{2} .
$$

Multiplying both sides of (24) by $h / 2$ and letting $j=m$ in (24), then after combining it with (25), we get

$$
\|u\|^{2} \leq \frac{1}{2}\left\|\delta_{x} u\right\|^{2}
$$

Similarly, we have

$$
\|u\|^{2} \leq \frac{1}{2}\left\|\delta_{y} u\right\|^{2}
$$

Combining (26) and (27), it follows

$$
\|u\| \leq \frac{1}{2}|u|_{1} .
$$

This completes the proof.

\section{Derivation of the Finite Difference Scheme}

Define the grid function $W=\left\{W_{i j}^{k} \mid 0 \leq i, j \leq m, 0 \leq k \leq n\right\}$ by

$$
W_{i j}^{k}=w\left(x_{i}, y_{j}, t_{k}\right), \quad 0 \leq i, j \leq m, 0 \leq k \leq n .
$$

Consider equation (1) at the inner points $\left(x_{i}, y_{j}, t_{k}\right)$ in $\Omega_{h \tau}$, then it follows

$$
\begin{gathered}
\frac{\partial^{2} w\left(x_{i}, y_{j}, t_{k}\right)}{\partial t^{2}}-\frac{\partial^{2} w\left(x_{i}, y_{j}, t_{k}\right)}{\partial x^{2}}-\frac{\partial^{2} w\left(x_{i}, y_{j}, t_{k}\right)}{\partial y^{2}}=f\left(x_{i}, y_{j}, t_{k}\right), \\
1 \leq i, j \leq m-1,1 \leq k \leq n-1 .
\end{gathered}
$$

According to Lemma 1(e), it follows

$$
\begin{aligned}
\delta_{t}^{2} W_{i j}^{k}-\delta_{x}^{2} W_{i j}^{\bar{k}}-\delta_{y}^{2} W_{i j}^{\bar{k}}= & f\left(x_{i}, y_{j}, t_{k}\right)+R_{i j}^{k}, \quad 1 \leq i, j \leq m \\
& -1,1 \leq k \leq n-1,
\end{aligned}
$$

where $R_{i j}^{k}=O\left(\tau^{2}+h^{2}\right)$. These are discrete equations at inner points.

Next we will pay our main attention to the derivation of difference equations at the boundary points. Considering the boundary condition 1.3 at $\left(x_{m}, y_{j}, t_{k}\right)$, it follows

$$
\frac{\partial w\left(x_{m}, y_{j}, t_{k}\right)}{\partial t}+\frac{\partial w\left(x_{m}, y_{j}, t_{k}\right)}{\partial x}=0, \quad 1 \leq j \leq m-1 .
$$

Similarly, the boundary condition 1.4 at $\left(x_{i}, y_{m}, t_{k}\right)$ satisfies

$$
\frac{\partial w\left(x_{i}, y_{m}, t_{k}\right)}{\partial t}+\frac{\partial w\left(x_{i}, y_{m}, t_{k}\right)}{\partial y}=0, \quad 1 \leq i \leq m-1
$$

By (3) and (4), the differential equation at the corner point $\left(x_{m}, y_{m}, t_{k}\right)$ reads

$$
\frac{2 \partial w\left(x_{m}, y_{m}, t_{k}\right)}{\partial t}+\frac{\partial w\left(x_{m}, y_{m}, t_{k}\right)}{\partial x}+\frac{\partial w\left(x_{m}, y_{m}, t_{k}\right)}{\partial y}=0
$$

According to Lemma 1 (a), (c), and (d), we have

$$
\begin{aligned}
\frac{\partial w\left(x_{m}, y_{j}, t_{k}\right)}{\partial t}= & \frac{W\left(x_{m}, y_{j}, t_{k+1}\right)-W\left(x_{m}, y_{j}, t_{k-1}\right)}{2 \tau} \\
& +O\left(\tau^{2}\right)=D_{t}^{-} W_{m j}^{k}+O\left(\tau^{2}\right)
\end{aligned}
$$

$$
\begin{aligned}
\frac{\partial w\left(x_{i}, y_{m}, t_{k}\right)}{\partial t} & =\frac{W\left(x_{i}, y_{m}, t_{k+1}\right)-W\left(x_{i}, y_{m}, t_{k-1}\right)}{2 \tau}+O\left(\tau^{2}\right) \\
& =D_{t} W_{i m}^{k}+O\left(\tau^{2}\right),
\end{aligned}
$$

$$
\begin{aligned}
\frac{\partial w\left(x_{m}, y_{j}, t_{k}\right)}{\partial x}= & \frac{1}{2}\left(\frac{\partial w\left(x_{m}, y_{j}, t_{k-1}\right)}{\partial x}+\frac{\partial w\left(x_{m}, y_{j}, t_{k+1}\right)}{\partial x}\right) \\
& +O\left(\tau^{2}\right) \\
= & D_{x-} W_{m j}^{\bar{k}}+\frac{h}{2}\left(\delta_{t}^{2} W_{m j}^{k}-\delta_{y}^{2} W_{m j}^{\bar{k}}\right. \\
& \left.-f\left(x_{m}, y_{j}, t_{k}\right)\right)+O\left(\tau^{2}+h^{2}\right),
\end{aligned}
$$

$$
\begin{aligned}
\frac{\partial w\left(x_{i}, y_{m}, t_{k}\right)}{\partial y}= & \frac{1}{2}\left(\frac{\partial w\left(x_{i}, y_{m}, t_{k-1}\right)}{\partial y}+\frac{\partial w\left(x_{i}, y_{m}, t_{k+1}\right)}{\partial y}\right) \\
& +O\left(\tau^{2}\right) \\
= & D_{y-} W_{i m}^{\bar{k}}+\frac{h}{2}\left(\delta_{t}^{2} W_{i m}^{k}-\delta_{x}^{2} W_{i m}^{\bar{k}}\right. \\
& \left.-f\left(x_{i}, y_{m}, t_{k}\right)\right)+O\left(\tau^{2}+h^{2}\right) .
\end{aligned}
$$

Applying (35) and (37) into (32), we get

$$
\begin{aligned}
& D_{x-} W_{m j}^{\bar{k}}+D_{t}^{-} W_{m j}^{k}+\frac{h}{2} \delta_{t}^{2} W_{m j}^{k}-\frac{h}{2} \delta_{y}^{2} W_{m j}^{\bar{k}}=\frac{h}{2} f\left(x_{m}, y_{j}, t_{k}\right) \\
& \quad+R_{m j}^{k}, \quad 1 \leq j \leq m-1,1 \leq k \leq n-1,
\end{aligned}
$$

where $R_{m j}^{k}=O\left(\tau^{2}+h^{2}\right)$. Similarly, we have 


$$
\begin{aligned}
& D_{y-} W_{i m}^{\bar{k}}+D_{t}^{2} W_{i m}^{k}+\frac{h}{2} \delta_{t}^{2} W_{i m}^{k}-\frac{h}{2} \delta_{x}^{2} W_{i m}^{\bar{k}}=\frac{h}{2} f\left(x_{i}, y_{m}, t_{k}\right)+R_{i m}^{k} \\
& D_{x-} W_{m m}^{\bar{k}}+D_{y-} W_{m m}^{\bar{k}}+2 D_{t} W_{m m}^{k}+\frac{h}{2} \delta_{t}^{2} W_{m m}^{k} \\
& =h f\left(x_{m}, y_{m}, t_{k}\right)+R_{m m}^{k}, \quad 1 \leq i \leq m-1,1 \leq k \leq n-1,
\end{aligned}
$$

where $R_{i m}^{k}=O\left(\tau^{2}+h^{2}\right)$ and $R_{m m}^{k}=O\left(\tau^{2}+h^{2}\right)$.

When $k=0$, equation (1) at initial grids $\left(x_{i}, y_{j}, t_{0}\right)$ satisfies

$$
\begin{array}{r}
\frac{\partial^{2} w\left(x_{i}, y_{j}, t_{0}\right)}{\partial t^{2}}-\frac{\partial^{2} w\left(x_{i}, y_{j}, t_{0}\right)}{\partial x^{2}}-\frac{\partial^{2} w\left(x_{i}, y_{j}, t_{0}\right)}{\partial y^{2}}= \\
f\left(x_{i}, y_{j}, t_{0}\right), \\
1 \leq i, j \leq m-1 .
\end{array}
$$

From Lemma 1(f) we have,

$$
\frac{\partial^{2} w\left(x_{i}, y_{j}, t_{0}\right)}{\partial t^{2}}=\frac{2}{\tau}\left(\delta_{t} W_{i j}^{1 / 2}-w_{t}\left(x_{i}, y_{j}, t_{0}\right)\right)+O(\tau) \text {. }
$$

Putting (43) into (42), then by Lemma 1(a) and (e), the following equations hold:

$$
\begin{aligned}
& \frac{2}{\tau}\left(\delta_{t} W_{i j}^{1 / 2}-w_{t}\left(x_{i}, y_{j}, t_{0}\right)\right)-\delta_{x}^{2} W_{i j}^{1 / 2}-\delta_{y}^{2} W_{i j}^{1 / 2}=f\left(x_{i}, y_{j}, t_{0}\right) \\
& \quad+r_{i j}, \quad 1 \leq i, j \leq m-1,
\end{aligned}
$$

where $r_{i j}=O\left(\tau+h^{2}\right)$. At $\left(x_{m}, y_{j}, t_{1 / 2}\right)$, we construct the following difference scheme:

$$
\begin{aligned}
\delta_{x} W_{m-(1 / 2), j}^{1 / 2}+\delta_{t} W_{m j}^{1 / 2}+\frac{h}{\tau} \delta_{t} W_{m j}^{1 / 2}-\frac{h}{2} \delta_{y}^{2} W_{m j}^{1 / 2}=\frac{h}{\tau} \psi\left(x_{m}, y_{j}\right) \\
+\frac{h}{2} f\left(x_{m}, y_{j}, t_{0}\right)+r_{m j},
\end{aligned}
$$

where $r_{m j}=O\left(\tau^{2}+h^{2}\right)$. Similarly, at $\left(x_{i}, y_{m}, t_{1 / 2}\right)$ and $\left(x_{m}, y_{m}, t_{1 / 2}\right)$, we have

$$
\begin{aligned}
& \delta_{y} W_{i, m-(1 / 2)}^{1 / 2}+\delta_{t} W_{i m}^{1 / 2}+\frac{h}{\tau} \delta_{t} W_{i m}^{1 / 2}-\frac{h}{2} \delta_{x}^{2} W_{i m}^{1 / 2}=\frac{h}{\tau} \psi\left(x_{i}, y_{m}\right) \\
& +\frac{h}{2} f\left(x_{i}, y_{m}, t_{0}\right)+r_{i m}
\end{aligned}
$$

$$
\begin{aligned}
& \delta_{x} W_{m-(1 / 2), m}^{1 / 2}+\delta_{y} W_{m, m-(1 / 2)}^{1 / 2}+2 \delta_{t} W_{m m}^{1 / 2}+\frac{h}{\tau} \delta_{t} W_{m m}^{1 / 2} \\
& =\frac{2 h}{\tau} \psi\left(x_{m}, y_{m}\right)+h f\left(x_{m}, y_{m}, t_{0}\right)+r_{m m}
\end{aligned}
$$

respectively, where $r_{i m}=O\left(\tau^{2}+h^{2}\right)$ and $r_{m m}=O\left(\tau^{2}+h^{2}\right)$. For the initial condition and the left and bottom boundaries, we have

$$
\begin{gathered}
W_{i j}^{0}=\varphi\left(x_{i}, y_{j}\right), \quad 0 \leq i, j \leq m, \\
W_{0 j}^{k}=W_{i 0}^{k}=0, \quad 0 \leq i \leq m, 1 \leq j \leq m, 1 \leq k \leq n .
\end{gathered}
$$

Finally, by dropping the infinitesimals in (31), (39)-(47), with $W_{i j}^{k}$ replaced by $w_{i j}^{k}$, we get the finite difference schemes for (1)-(4) as follows

$$
\begin{array}{r}
\delta_{t}^{2} w_{i j}^{k}-\delta_{x}^{2} w_{i j}^{\bar{k}}-\delta_{y}^{2} w_{i j}^{\bar{k}}=f\left(x_{i}, y_{j}, t_{k}\right), \quad 1 \leq i, j \leq m-1, \\
1 \leq k \leq n-1,
\end{array}
$$

$$
D_{x-} w_{m j}^{\bar{k}}+D_{t} w_{m j}^{k}+\frac{h}{2} \delta_{t}^{2} w_{m j}^{k}-\frac{h}{2} \delta_{y}^{2} w_{m j}^{\bar{k}}=\frac{h}{2} f\left(x_{m}, y_{j}, t_{k}\right),
$$$$
1 \leq j \leq m-1,1 \leq k \leq n-1 \text {, }
$$

$$
\begin{gathered}
D_{y-} w_{i m}^{\bar{k}}+D_{t}^{-} w_{i m}^{k}+\frac{h}{2} \delta_{t}^{2} w_{i m}^{k}-\frac{h}{2} \delta_{x}^{2} w_{i m}^{\bar{k}}=\frac{h}{2} f\left(x_{i}, y_{m}, t_{k}\right), \\
1 \leq j \leq m-1,1 \leq k \leq n-1,
\end{gathered}
$$

$$
\begin{gathered}
D_{x-} w_{m m}^{\bar{k}}+D_{y-} w_{m m}^{\bar{k}}+2 D_{\bar{t}} w_{m m}^{k}+\frac{h}{2} \delta_{t}^{2} w_{m m}^{k}=\frac{h}{2} f\left(x_{m}, y_{m}, t_{k}\right), \\
1 \leq k \leq n-1,
\end{gathered}
$$

$$
\begin{gathered}
\frac{2}{\tau} \delta_{t} w_{i j}^{1 / 2}-\delta_{x}^{2} w_{i j}^{1 / 2}-\delta_{y}^{2} w_{i j}^{1 / 2}=f\left(x_{i}, y_{j}, t_{0}\right)+\frac{2}{\tau} \psi\left(x_{i}, y_{j}\right) \\
1 \leq i, j \leq m-1, \\
\delta_{x} w_{m-(1 / 2), j}^{1 / 2}+\delta_{t} w_{m j}^{1 / 2}+\frac{h}{\tau} \delta_{t} w_{m j}^{1 / 2}-\frac{h}{2} \delta_{y}^{2} w_{m j}^{1 / 2}=\frac{h}{\tau} \psi\left(x_{m}, y_{j}\right) \\
+\frac{h}{2} f\left(x_{m}, y_{j}, t_{0}\right), \quad 1 \leq j \leq m-1,
\end{gathered}
$$

$$
\begin{aligned}
& \delta_{y} w_{i, m-(1 / 2)}^{1 / 2}+\delta_{t} w_{i m}^{1 / 2}+\frac{h}{\tau} \delta_{t} w_{i m}^{1 / 2}-\frac{h}{2} \delta_{x}^{2} w_{i m}^{1 / 2}=\frac{h}{\tau} \psi\left(x_{i}, y_{m}\right) \\
& +\frac{h}{2} f\left(x_{i}, y_{m}, t_{0}\right), \quad 1 \leq i \leq m-1,
\end{aligned}
$$

$$
\begin{aligned}
& \delta_{x} w_{m-(1 / 2), m}^{1 / 2}+\delta_{y} w_{m, m-(1 / 2)}^{1 / 2}+2 \delta_{t} w_{m m}^{1 / 2}+\frac{h}{\tau} \delta_{t} w_{m m}^{1 / 2} \\
& =\frac{2 h}{\tau} \psi\left(x_{m}, y_{m}\right)+\frac{h}{2} f\left(x_{m}, y_{m}, t_{0}\right),
\end{aligned}
$$




$$
\begin{aligned}
w_{i j}^{0} & =\varphi\left(x_{i}, y_{j}\right), \quad 0 \leq i, j \leq m, \\
w_{i 0}^{k} & =0, \\
w_{0 j}^{k} & =0, \\
0 & \leq i \leq m, 1 \leq j \leq m, 1 \leq k \leq n .
\end{aligned}
$$

\section{A Priori Estimate of the Difference Scheme}

In order to prove the convergence and stability of difference schemes, we give a priori estimate of difference schemes (50)-(59).

Theorem 1. Suppose that $\left\{u_{i j}^{k} \mid 0 \leq i, j \leq m, 0 \leq k \leq n\right\}$ solves the following difference scheme:

$$
\begin{aligned}
& \delta_{t}^{2} u_{i j}^{k}-\delta_{x}^{2} u_{i j}^{\bar{k}}-\delta_{y}^{2} u_{i j}^{\bar{k}}=f_{i j}^{k}, \quad 1 \leq i, j \leq m-1,1 \leq k \leq n-1, \\
& D_{x-} u_{m j}^{\bar{k}}+D_{t} u_{m j}^{k}+\frac{h}{2} \delta_{t}^{2} u_{m j}^{k}-\frac{h}{2} \delta_{y}^{2} u_{m j}^{\bar{k}}=f_{m j}^{k}, \quad 1 \leq j \leq m-1, \\
& 1 \leq k \leq n-1, \\
& D_{y-} u_{i m}^{\bar{k}}+D_{t} u_{i m}^{k}+\frac{h}{2} \delta_{t}^{2} u_{i m}^{k}-\frac{h}{2} \delta_{x}^{2} u_{i m}^{\bar{k}}=f_{i m}^{k}, \quad 1 \leq i \leq m-1, \\
& 1 \leq k \leq n-1,
\end{aligned}
$$

$$
D_{x-} u_{m m}^{\bar{k}}+D_{y-} u_{m m}^{\bar{k}}+2 D_{t} u_{m m}^{k}+\frac{h}{2} \delta_{t}^{2} u_{m m}^{k}=f_{m m}^{k}, \quad 1 \leq k \leq n-1,
$$

$$
\frac{2}{\tau} \delta_{t} u_{i j}^{1 / 2}-\delta_{x}^{2} u_{i j}^{1 / 2}-\delta_{y}^{2} u_{i j}^{1 / 2}=f_{i j}^{0}, \quad 1 \leq i, j \leq m-1
$$

$$
\delta_{x} u_{m-(1 / 2), j}^{1 / 2}+\delta_{t} u_{m j}^{1 / 2}+\frac{h}{\tau} \delta_{t} u_{m j}^{1 / 2}-\frac{h}{2} \delta_{y}^{2} u_{m j}^{1 / 2}=f_{m j}^{0}, \quad 1 \leq j \leq m-1,
$$

$$
\delta_{y} u_{i, m-(1 / 2)}^{1 / 2}+\delta_{t} u_{i m}^{1 / 2}+\frac{h}{\tau} \delta_{t} u_{i m}^{1 / 2}-\frac{h}{2} \delta_{x}^{2} u_{i m}^{1 / 2}=f_{i m}^{0}, \quad 1 \leq i \leq m-1
$$

$$
\delta_{x} u_{m-(1 / 2), m}^{1 / 2}+\delta_{y} u_{m, m-(1 / 2)}^{1 / 2}+2 \delta_{t} u_{m m}^{1 / 2}+\frac{h}{\tau} \delta_{t} u_{m m}^{1 / 2}=f_{m m}^{0}
$$

$$
\begin{aligned}
u_{i j}^{0} & =\varphi\left(x_{i}, y_{j}\right), \quad 0 \leq i, j \leq m, \\
u_{i 0}^{k} & =0, \\
u_{0 j}^{k} & =0, \\
0 & \leq i \leq m, 1 \leq j \leq m, 1 \leq k \leq n,
\end{aligned}
$$

then for arbitrary grid ratio $\lambda=\tau / h$, we have

$$
\begin{array}{r}
\left\|\delta_{t} u^{k+(1 / 2)}\right\|^{2}+\frac{1}{2}\left(\left|u^{k+1}\right|_{1}^{2}+\left|u^{k}\right|_{1}^{2}\right) \leq \exp \left(\frac{3}{2} T\right)\left(|\varphi|_{1}^{2}+\frac{\tau^{2}}{4}\left\|f^{0}\right\|^{2}+\frac{h \tau}{4} \sum_{j=1}^{m-1}\left|f_{m j}^{0}\right|^{2}+\frac{h \tau}{4} \sum_{i=1}^{m-1}\left|f_{i m}^{0}\right|^{2}+\frac{h \tau}{16}\left|f_{m m}^{0}\right|^{2}+\frac{3 \tau}{2} \sum_{l=1}^{k} G^{l}\right) \\
1 \leq k \leq n-1
\end{array}
$$

where

$$
G^{l}=h^{2} \sum_{i, j=1}^{m-1}\left(f_{i j}^{k}\right)^{2}+\frac{h}{2} \sum_{j=1}^{m-1}\left|f_{m j}^{l}\right|^{2}+\frac{h}{2} \sum_{i=1}^{m-1}\left|f_{i m}^{l}\right|^{2}+\frac{h}{8}\left|f_{m m}^{l}\right|^{2}, \quad 1 \leq l \leq n-1 .
$$

Proof. Define

$$
E^{k}=\left\|\delta_{t} u^{k+(1 / 2)}\right\|^{2}+\frac{1}{2}\left(\left|u^{k+1}\right|_{1}^{2}+\left|u^{k}\right|_{1}^{2}\right) .
$$

Multiplying both sides of (60) by $2 h^{2} D_{t} u_{i j}^{k}$, and taking summation for $i$ and $j$ from 1 to $m-1$, we get 


$$
2 h^{2} \sum_{i, j=1}^{m-1} \delta_{t}^{2} u_{i j}^{k} D_{t}^{-} u_{i j}^{k}-2 h^{2} \sum_{i, j=1}^{m-1} \delta_{x}^{2} u_{i j}^{\bar{k}} D_{t}^{-} u_{i j}^{k}-2 h^{2} \sum_{i, j=1}^{m-1} \delta_{y}^{2} u_{i j}^{\bar{k}} D_{t}^{-} u_{i j}^{k}=2 h^{2} \sum_{i, j=1}^{m-1} f_{i j}^{k} D_{t} u_{i j}^{k}, \quad 1 \leq k \leq n-1
$$

The first term in (73) implies

$$
2 h^{2} \sum_{i, j=1}^{m-1} \delta_{t}^{2} u_{i, j}^{k} D_{t} u_{i j}^{k}=\frac{1}{\tau}\left(h^{2} \sum_{i, j=1}^{m-1}\left(\delta_{t} u_{i j}^{k+(1 / 2)}\right)^{2}-h^{2} \sum_{i, j=1}^{m-1}\left(\delta_{t} u_{i j}^{k-(1 / 2)}\right)^{2}\right) \text {. }
$$

$$
\begin{aligned}
- & 2 h^{2} \sum_{i, j=1}^{m-1} \delta_{x}^{2} u_{i j}^{\bar{k}} D_{t} u_{i j}^{k} \\
= & -2 h \sum_{i, j=1}^{m-1}\left(\delta_{x} u_{i+(1 / 2), j}^{\bar{k}}-\delta_{x} u_{i-(1 / 2), j}^{\bar{k}}\right) D_{t} u_{i j}^{k} \\
= & 2 h^{2} \sum_{i=0}^{m-1} \sum_{j=1}^{m-1} \delta_{x} u_{i+(1 / 2), j}^{\bar{k}} D_{t} \delta_{x} u_{i+(1 / 2), j}^{k}-2 h \sum_{j=1}^{m-1} D_{x-} u_{m j}^{\bar{k}} D_{t} u_{m j}^{k} \\
= & \frac{1}{2 \tau}\left(h^{2} \sum_{i=0}^{m-1} \sum_{j=1}^{m-1}\left(\delta_{x} u_{i+(1 / 2), j}^{k+1}\right)^{2}-h^{2} \sum_{i=0}^{m-1} \sum_{j=1}^{m-1}\left(\delta_{x} u_{i+(1 / 2), j}^{k-1}\right)^{2}\right)-2 h \sum_{j=1}^{m-1} D_{x-} u_{m j}^{\bar{k}} D_{t} u_{m j}^{k}, \\
& -2 h^{2} \sum_{i, j=1}^{m-1} \delta_{y}^{2} u_{i j}^{\bar{k}} D_{t} u_{i j}^{k} \\
= & \frac{1}{2 \tau}\left(h^{2} \sum_{i=1}^{m-1} \sum_{j=0}^{m-1}\left(\delta_{y} u_{i, j+(1 / 2)}^{k+1}\right)^{2}-h^{2} \sum_{i=1}^{m-1} \sum_{j=0}^{m-1}\left(\delta_{y} u_{i, j+(1 / 2)}^{k-1}\right)^{2}\right)-2 h \sum_{i=1}^{m-1} D_{y-1} u_{i m}^{\bar{k}} D_{t}^{-} u_{i m}^{k},
\end{aligned}
$$

respectively. Putting (74), (75) and (76) into (73), we get

$$
\begin{aligned}
& \frac{1}{\tau}\left(h^{2} \sum_{i, j=1}^{m-1}\left(\delta_{t} u_{i j}^{k+(1 / 2)}\right)^{2}-h^{2} \sum_{i, j=1}^{m-1}\left(\delta_{t} u_{i j}^{k-(1 / 2)}\right)^{2}\right)+\frac{1}{2 \tau}\left(h^{2} \sum_{i=0}^{m-1} \sum_{j=1}^{m-1}\left(\delta_{x} u_{i+(1 / 2), j}^{k+1}\right)^{2}\right. \\
& \left.-h^{2} \sum_{i=0}^{m-1} \sum_{j=1}^{m-1}\left(\delta_{x} u_{i+(1 / 2), j}^{k-1}\right)^{2}\right)+\frac{1}{2 \tau}\left(h^{2} \sum_{i=1}^{m-1} \sum_{j=0}^{m-1}\left(\delta_{y} u_{i, j+(1 / 2)}^{k+1}\right)^{2}-h^{2} \sum_{i=1}^{m-1} \sum_{j=0}^{m-1}\left(\delta_{y} u_{i, j+(1 / 2)}^{k-1}\right)^{2}\right) \\
& =2 h^{2} \sum_{i, j=1}^{m-1} f_{i j}^{k} D_{t} u_{i j}^{k}+2 h \sum_{j=1}^{m-1} D_{x-} u_{m j}^{\bar{k}} D_{t}^{-} u_{m j}^{k}+2 h \sum_{i=1}^{m-1} D_{y-} u_{i m}^{k} D_{t} u_{i m}^{k},
\end{aligned}
$$


where

$$
\begin{aligned}
& 2 h^{2} \sum_{i, j=1}^{m-1} f_{i j}^{k} D_{t} u_{i j}^{k} \leq h^{2} \sum_{i, j=1}^{m-1}\left(D_{t} u_{i j}^{k}\right)^{2}+h^{2} \sum_{i, j=1}^{m-1}\left(f_{i j}^{k}\right)^{2} \\
& \leq \frac{1}{2}\left(h^{2} \sum_{i, j=1}^{m-1}\left(\delta_{t} u_{i j}^{k+(1 / 2)}\right)^{2}+h^{2} \sum_{i, j=1}^{m-1}\left(\delta_{t} u_{i j}^{k-(1 / 2)}\right)^{2}\right)+h^{2} \sum_{i, j=1}^{m-1}\left(f_{i j}^{k}\right)^{2} .
\end{aligned}
$$

Considering (61)-(63) and by inequality $2(b-a) a \leq(1 / 2) b^{2}$, we get

$$
\begin{aligned}
2 h & \sum_{j=1}^{m-1} D_{x-} u_{m j}^{\bar{k}} D_{t} u_{m j}^{k}+2 h \sum_{i=1}^{m-1} D_{y-} u_{i m}^{\bar{k}} D_{t} u_{i m}^{k} \\
= & h \sum_{j=1}^{m-1}\left(2\left(f_{m j}^{k}-D_{t} u_{m j}^{k}\right) D_{t} u_{m j}^{k}-h \delta_{t}^{2} u_{m j}^{k} D_{t} u_{m j}^{k}+h \delta_{y}^{2} u_{m j}^{\bar{k}} D_{t} u_{m j}^{k}\right) \\
& +h \sum_{i=1}^{m-1}\left(2\left(f_{i m}^{k}-D_{t} u_{i m}^{k}\right) D_{t} u_{i m}^{k}-h \delta_{t}^{2} u_{i m}^{k} D_{t} u_{i m}^{k}+h \delta_{x}^{2} u_{i m}^{\bar{k}} D_{t} u_{i m}^{k}\right) \\
\leq & -\frac{h^{2}}{2 \tau} \sum_{j=1}^{m-1}\left(\left(\delta_{t} u_{m j}^{k+(1 / 2)}\right)^{2}-\left(\delta_{t} u_{m j}^{k-(1 / 2)}\right)^{2}\right)-\frac{h^{2}}{4 \tau} \sum_{j=0}^{m-1}\left(\left(\delta_{y} u_{m, j+(1 / 2)}^{k+1}\right)^{2}-\left(\delta_{y} u_{m, j+(1 / 2)}^{k-1}\right)^{2}\right) \\
& -\frac{h^{2}}{2 \tau} \sum_{i=1}^{m-1}\left(\left(\delta_{t} u_{i m}^{k+(1 / 2)}\right)^{2}-\left(\delta_{t} u_{i m}^{k-(1 / 2)}\right)^{2}\right)-\frac{h^{2}}{4 \tau} \sum_{i=0}^{m-1}\left(\left(\delta_{x} u_{i+(1 / 2), m}^{k+1}\right)^{2}-\left(\delta_{x} u_{i+(1 / 2), m}^{k-1}\right)^{2}\right) \\
& +\frac{h^{\prime}}{8}\left|f_{m m}^{k}\right|^{2}-\frac{h^{2}}{4 \tau}\left(\left(\delta_{t} u_{m m}^{k+(1 / 2)}\right)^{2}-\left(\delta_{t} u_{m m}^{k-(1 / 2)}\right)^{2}\right)+\frac{h}{2} \sum_{j=1}^{m-1}\left|f_{m j}^{k}\right|^{2}+\frac{h}{2} \sum_{i=1}^{m-1}\left|f_{i m}^{k}\right|^{2} .
\end{aligned}
$$

According to the definition of $E^{k}$, (77) implies

$$
\begin{gathered}
\frac{1}{\tau}\left(E^{k}-E^{k-1}\right) \leq \frac{1}{2}\left(E^{k}+E^{k-1}\right)+h^{2} \sum_{i, j=1}^{m-1}\left(f_{i j}^{k}\right)^{2}+\frac{h}{2} \sum_{j=1}^{m-1}\left|f_{m j}^{k}\right|^{2} \\
+\frac{h}{2} \sum_{i=1}^{m-1}\left|f_{i m}^{k}\right|^{2}+\frac{h}{8}\left|f_{m m}^{k}\right|^{2}, \quad 1 \leq k \leq n-1 .
\end{gathered}
$$

If we confine $\tau \leq(2 / 3)$, then

$$
E^{k} \leq\left(1+\frac{3 \tau}{2}\right) E^{k-1}+\frac{3 \tau}{2} G^{k}, \quad 1 \leq k \leq n-1 .
$$

By Gronwall inequality in Lemma 2, we get

$$
E^{k} \leq \exp \left(\frac{3}{2} k \tau\right)\left(E^{0}+\frac{3 \tau}{2} \sum_{l=1}^{k} G^{l}\right), \quad 1 \leq k \leq n-1 .
$$

Next we estimate $E^{0}$. Multiplying (64) by $h^{2} \delta_{t} u_{i j}^{1 / 2}$, and summing up for $i$ and $j$ from 1 to $m-1$, we have

$$
\begin{aligned}
& \frac{2}{\tau} h^{2} \sum_{i, j=1}^{m-1}\left(\delta_{t} u_{i j}^{1 / 2}\right)^{2}-h^{2} \sum_{i, j=1}^{m-1}\left(\delta_{x}^{2} u_{i j}^{1 / 2}+\delta_{y}^{2} u_{i j}^{1 / 2}\right) \delta_{t} u_{i j}^{1 / 2} \\
& =h^{2} \sum_{i, j=1}^{m-1} f_{i j}^{0} \delta_{t} u_{i j}^{1 / 2}
\end{aligned}
$$

which similarly implies

$$
\begin{aligned}
& \frac{2}{\tau}\left\|\delta_{t} u^{1 / 2}\right\|^{2}+\frac{1}{2 \tau}\left(\left|u^{1}\right|_{1}^{2}-\left|u^{0}\right|_{1}^{2}\right) \\
& \leq \frac{1}{\tau}\left\|\delta_{t} u^{1 / 2}\right\|^{2}+\frac{\tau}{4}\left\|f^{0}\right\|^{2}+\frac{h}{4} \sum_{j=1}^{m-1}\left|f_{m j}^{0}\right|^{2}+\frac{h}{4} \sum_{i=1}^{m-1}\left|f_{i m}^{0}\right|^{2}+\frac{h}{16}\left|f_{m m}^{0}\right|^{2} .
\end{aligned}
$$


According to the definition of $E^{0}$, we obtain

$$
\begin{array}{r}
E^{0}=\left\|\delta_{t} u^{1 / 2}\right\|^{2}+\frac{1}{2}\left(\left|u^{1}\right|_{1}^{2}+\left|u^{0}\right|_{1}^{2}\right) \\
\leq\left|u^{0}\right|_{1}^{2}+\frac{\tau^{2}}{4}\left\|f^{0}\right\|^{2}+\frac{h \tau}{4} \sum_{j=1}^{m-1}\left|f_{m j}^{0}\right|^{2}+\frac{h \tau}{4} \sum_{i=1}^{m-1}\left|f_{i m}^{0}\right|^{2}+\frac{h \tau}{16}\left|f_{m m}^{0}\right|^{2} .
\end{array}
$$

Putting (85) into (82) and noticing $k \tau \leq T$, we achieve (70). This completes the proof.

\section{Existence, Convergence, and Stability}

In this section, we will discuss the unique solvability, convergence, and stability of the difference scheme (50)-(59).

Theorem 2. The difference scheme (50)-(59) is uniquely solvable.

Proof. Notice that $w^{0}$ is known by the initial condition. The difference scheme for $w^{1}$ is (54)-(59). We consider the following homogeneous equations of (54)-(59):

$$
\begin{gathered}
\frac{2}{\tau^{2}} w_{i j}^{1}-\frac{1}{2} \delta_{x}^{2} w_{i j}^{1}-\frac{1}{2} \delta_{y}^{2} w_{i j}^{1}=0, \quad 1 \leq i, j \leq m-1, \\
\frac{1}{2} \delta_{x} w_{m-(1 / 2), j}^{1}+\frac{1}{\tau} w_{m j}^{1}+\frac{h}{\tau^{2}} w_{m j}^{1}-\frac{h}{4} \delta_{y}^{2} w_{m j}^{1}=0, \quad 1 \leq j \leq m-1,
\end{gathered}
$$

$$
\frac{1}{2} \delta_{y} w_{i, m-(1 / 2)}^{1}+\frac{1}{\tau} w_{i m}^{1}+\frac{h}{\tau^{2}} w_{i m}^{1}-\frac{h}{4} \delta_{x}^{2} w_{i m}^{1}=0, \quad 1 \leq i \leq m-1,
$$

$$
\begin{gathered}
\frac{1}{2} \delta_{x} w_{m-(1 / 2), m}^{1}+\frac{1}{2} \delta_{y} w_{m, m-(1 / 2)}^{1}+\frac{2}{\tau} w_{m m}^{1}+\frac{h}{\tau^{2}} w_{m m}^{1}=0, \\
w_{i 0}^{1}=0, \quad 0 \leq i \leq m, \\
w_{0 j}^{1}=0, \quad 1 \leq j \leq m .
\end{gathered}
$$

Multiplying (86) by $h^{2} w_{i j}^{1}$, summing up for $i$ and $j$ from 1 to $m-1$, and combining with (87)-(90), we get

$$
\frac{2}{\tau^{2}}\left\|w^{1}\right\|^{2}+\frac{1}{2}\left|w^{1}\right|_{1}^{2}+\frac{h}{\tau}\left(\sum_{j=1}^{m-1}\left(w_{m j}^{1}\right)^{2}+\sum_{i=1}^{m-1}\left(w_{i m}^{1}\right)^{2}+\left(w_{m m}^{1}\right)^{2}\right)=0,
$$

which implies $w_{i j}^{1}=0(0 \leq i, j \leq m)$. Thus, $w^{1}$ is uniquely solvable.

Now suppose that $w^{k-1}$ and $w^{k}$ are uniquely solvable. The difference schemes for $w^{k+1}$ satisfy (50)-(53) and (59). The homogeneous equations read:

$$
\begin{gathered}
\frac{1}{\tau^{2}} w_{i j}^{k+1}-\frac{1}{2} \delta_{x}^{2} w_{i j}^{k+1}-\frac{1}{2} \delta_{y}^{2} w_{i j}^{k+1}=0, \quad 1 \leq i, j \leq m-1,1 \leq k \leq n-1, \\
\frac{1}{2} D_{x-} w_{m j}^{k+1}+\frac{1}{2 \tau} w_{m j}^{k+1}+\frac{h}{2 \tau^{2}} w_{m j}^{k+1}-\frac{h}{4} \delta_{y}^{2} w_{m j}^{k+1}=0, \\
1 \leq j \leq m-1,1 \leq k \leq n-1, \\
\frac{1}{2} D_{y-} w_{i m}^{k+1}+\frac{1}{2 \tau} w_{i m}^{k+1}+\frac{h}{2 \tau^{2}} w_{i m}^{k+1}-\frac{h}{4} \delta_{x}^{2} w_{i m}^{k+1}=0, \\
1 \leq i \leq m-1,1 \leq k \leq n-1, \\
\frac{1}{2} D_{x-} w_{m m}^{k+1}+\frac{1}{2} D_{y-} w_{m m}^{k+1}+\frac{1}{\tau} w_{m m}^{k+1}+\frac{h}{2 \tau^{2}} w_{m m}^{k+1}=0, \\
\quad 1 \leq k \leq n-1, \\
w_{i 0}^{k}=0, \\
w_{0 j}^{k}=0, \\
0 \leq i \leq m, 0 \leq j \leq m, 1 \leq k \leq n .
\end{gathered}
$$

Multiplying (92) by $h^{2} w_{i j}^{k+1}$, and summing up for $i$ and $j$ from 1 to $m-1$, and applying equations (93)-(96), we have

$$
\frac{1}{\tau^{2}}\left\|w^{k+1}\right\|^{2}+\frac{1}{2}\left|w^{k+1}\right|_{1}^{2}+\frac{h}{2 \tau}\left(\sum_{j=1}^{m-1}\left(w_{m j}^{k+1}\right)^{2}+\sum_{i=1}^{m-1}\left(w_{i m}^{k+1}\right)^{2}+\left(w_{m m}^{k+1}\right)^{2}\right)=0,
$$

which means that $w_{i j}^{k+1}=0(0 \leq i, j \leq m)$. Thus $w^{k+1}$ is uniquely solvable.

According to the principle of induction, the difference scheme of (50)-(59) is uniquely solvable for all $w^{k}(1 \leq k \leq n)$. This completes the proof.

Define the pointwise error by

$$
e_{i j}^{k}=W_{i j}^{k}-w_{i j}^{k}, \quad 0 \leq i, j \leq m, 0 \leq k \leq n .
$$

The following theorem states the convergence of difference scheme (50)-(59).

Theorem 3. Suppose that $w(x, y, t) \in C_{x, y, t}^{4,4,3}(\bar{\Omega} \times[0, T]$ is the solution of (1)-(4) and $\left\{w_{i j}^{k} \mid 0 \leq i, j \leq m, 0 \leq k \leq n\right\}$ is the solution of difference scheme (50)-(59). Then there exists a constant $C_{T}$ such that

$$
\left\|\delta_{t} e^{k+(1 / 2)}\right\|^{2}+\frac{1}{2}\left(\left|e^{k+1}\right|_{1}^{2}+\left|e^{k}\right|_{1}^{2}\right) \leq C_{T}\left(\tau^{2}+h^{2}\right)^{2}, \quad 0 \leq k \leq n-1,
$$

where $C_{T}=\exp ((3 / 2) T)((5 / 4)+(3 / 2) T) c^{2}$.

Proof. Subtracting (50)-(59) from (31), (39)-(41) and (44)-(49) respectively, we have the following error equation 


$$
\begin{aligned}
& \delta_{t}^{2} e_{i j}^{k}-\delta_{x}^{2} e_{i j}^{\bar{k}}-\delta_{y}^{2} e_{i j}^{\bar{k}}=R_{i j}^{k}, \quad 1 \leq i, j \leq m-1,1 \leq k \leq n-1, \\
& D_{x-} e_{m j}^{\bar{k}}+D_{t} e_{m j}^{k}+\frac{h}{2} \delta_{t}^{2} e_{m j}^{k}-\frac{h}{2} \delta_{y}^{2} e_{m j}^{\bar{k}}=R_{m j}^{k}, \quad 1 \leq j \leq m-1,1 \leq k \leq n-1, \\
& D_{y-} e_{i m}^{\bar{k}}+D_{t} e_{i m}^{k}+\frac{h}{2} \delta_{t}^{2} e_{i m}^{k}-\frac{h}{2} \delta_{x}^{2} e_{i m}^{\bar{k}}=R_{i m}^{k}, \quad 1 \leq i \leq m-1,1 \leq k \leq n-1, \\
& D_{x-} e_{m m}^{\bar{k}}+D_{y-} e_{m m}^{\bar{k}}+2 D_{t} e_{m m}^{k}+\frac{h}{2} \delta_{t}^{2} e_{m m}^{k}=R_{m m}^{k}, \quad 1 \leq k \leq n-1, \\
& \frac{2}{\tau} \delta_{t} e_{i j}^{1 / 2}-\delta_{x}^{2} e_{i j}^{1 / 2}-\delta_{y}^{2} e_{i j}^{1 / 2}=r_{i j}, \quad 1 \leq i, j \leq m-1, \\
& \delta_{x} e_{m-(1 / 2), j}^{1 / 2}+\delta_{t} e_{m j}^{1 / 2}+\frac{h}{\tau} \delta_{t} e_{m j}^{1 / 2}-\frac{h}{2} \delta_{y}^{2} e_{m j}^{1 / 2}=r_{m j}, \quad 1 \leq j \leq m-1, \\
& \delta_{y} e_{i, m-(1 / 2)}^{1 / 2}+\delta_{t} e_{i m}^{1 / 2}+\frac{h}{\tau} \delta_{t} e_{i m}^{1 / 2}-\frac{h}{2} \delta_{x}^{2} e_{i m}^{1 / 2}=r_{i m}, \quad 1 \leq i \leq m-1, \\
& \delta_{x} e_{m-(1 / 2), m}^{1 / 2}+\delta_{y} e_{m, m-(1 / 2)}^{1 / 2}+2 \delta_{t} e_{m m}^{1 / 2}+\frac{h}{\tau} \delta_{t} e_{m m}^{1 / 2}=r_{m m}, \\
& e_{i j}^{0}=0, \quad 0 \leq i, j \leq m, \\
& e_{0 j}^{k}=e_{i 0}^{k}=0, \quad 0 \leq i \leq m, 1 \leq j \leq m, 0 \leq k \leq n .
\end{aligned}
$$

According to Theorem 1, we get

$$
\begin{aligned}
\left\|\delta_{t} e^{k+(1 / 2)}\right\|^{2}+\frac{1}{2}\left(\left|e^{k+1}\right|_{1}^{2}+\left|e^{k}\right|_{1}^{2}\right) \leq & \exp \left(\frac{3}{2} k \tau\right)\left(\left|e^{0}\right|_{1}^{2}+\frac{\tau^{2}}{4}\|r\|^{2}+\frac{h \tau}{4} \sum_{j=1}^{m-1}\left|r_{m j}\right|^{2}\right. \\
& \left.+\frac{h \tau}{4} \sum_{i=1}^{m-1}\left|r_{i m}\right|^{2}+\frac{h \tau}{16}\left|r_{m m}\right|^{2}+\frac{3 \tau}{2} \sum_{l=1}^{k} \widehat{G}^{l}\right), \quad 1 \leq k \leq n-1,
\end{aligned}
$$

where

$\widehat{G}^{l}=\left\|R^{l}\right\|^{2}+\frac{h}{2} \sum_{j=1}^{m-1}\left|R_{m j}^{l}\right|^{2}+\frac{h}{2} \sum_{i=1}^{m-1}\left|R_{i m}^{l}\right|^{2}+\frac{h}{8}\left|R_{m m}^{l}\right|^{2}, \quad 1 \leq l \leq n-1$.

(102)
Taking into account the order of $r_{i j}$ and $R_{i j}^{k}(0 \leq i, j \leq m, 1 \leq k \leq n)$, and the fact that $e_{i j}^{0}=0$, there exists a generic constant $c$ such that

$$
\begin{aligned}
& \left\|\delta_{t} e^{k+(1 / 2)}\right\|^{2}+\frac{1}{2}\left(\left|e^{k+1}\right|_{1}^{2}+\left|e^{k}\right|_{1}^{2}\right) \\
& \leq \exp \left(\frac{3}{2} k \tau\right)\left(\frac{\tau^{2}}{4} c^{2}\left(\tau+h^{2}\right)^{2}+\frac{\tau h}{4} c^{2}\left(\tau^{2}+h^{2}\right)^{2}+\frac{\tau h}{4} c^{2}\left(\tau^{2}+h^{2}\right)^{2}+\frac{\tau h}{16} c^{2}\left(\tau^{2}+h^{2}\right)^{2}+\frac{3}{2} k \tau c^{2}\left(\tau^{2}+h^{2}\right)^{2}\right) \\
& \leq \exp \left(\frac{3}{2} T\right)\left(\frac{5}{4}+\frac{3}{2} T\right) c^{2}\left(\tau^{2}+h^{2}\right)^{2}=C_{T}\left(\tau^{2}+h^{2}\right)^{2}, \quad 0 \leq k \leq n-1 .
\end{aligned}
$$




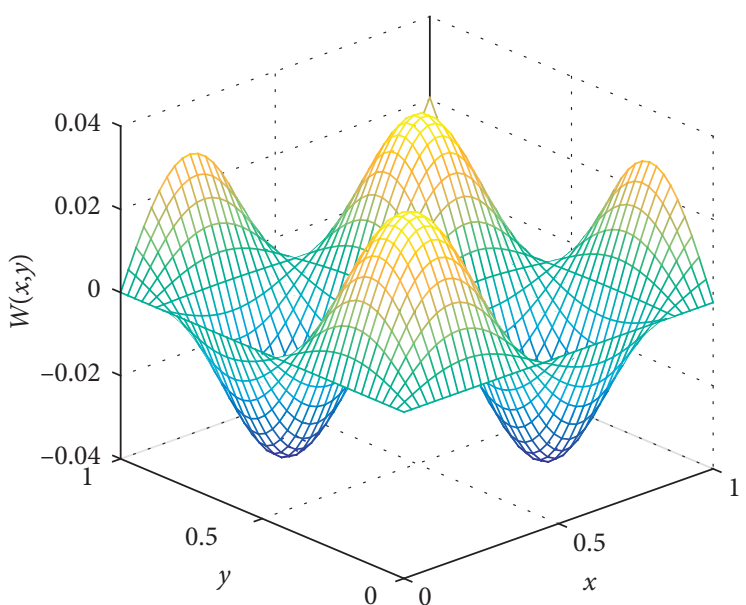

(a)

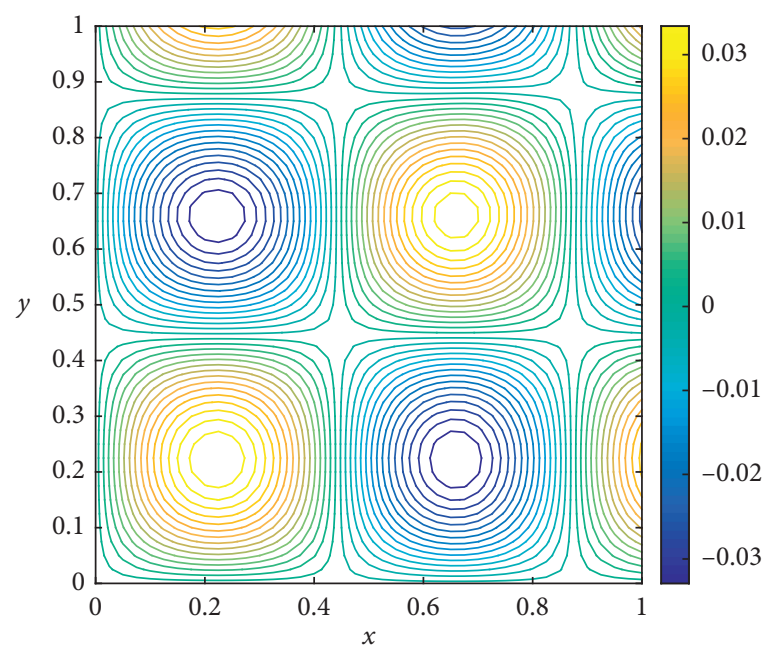

(b)

Figure 1: The evolution mesh (a) and contour map (b) of numerical solution.

TABle 1: Numerical convergence of scheme (50)-(59) in time $(h=1 / 100, T=1)$.

\begin{tabular}{lcc}
\hline$\tau$ & $E W(\tau, h)$ & Order \\
\hline $1 / 20$ & $1.2948 e-002$ & - \\
$1 / 40$ & $2.9438 e-003$ & 2.1370 \\
$1 / 50$ & $1.8099 e-003$ & 2.1799 \\
$1 / 60$ & $1.2057 e-003$ & 2.2280 \\
$1 / 70$ & $8.4660 e-004$ & 2.2938 \\
\hline
\end{tabular}

TABLe 2: Numerical convergence of scheme (50)-(59) in space $(\tau=1 / 200, T=1)$.

\begin{tabular}{lcc}
\hline$h$ & $E W(\tau, h)$ & Order \\
\hline $1 / 20$ & $2.4487 e-003$ & - \\
$1 / 30$ & $1.0292 e-003$ & 2.1377 \\
$1 / 40$ & $5.4095 e-004$ & 2.2358 \\
$1 / 50$ & $3.1889 e-004$ & 2.3683 \\
$1 / 60$ & $2.0234 e-004$ & 2.4950 \\
\hline
\end{tabular}

This completes the proof.

According to Lemma 4, we can conclude that $\left\|e^{k}\right\|=O\left(\tau^{2}+h^{2}\right)(1 \leq k \leq n)$, namely, the difference solution is of second-order convergence in $L^{2}$ norm.
TABLE 3: Numerical convergence of scheme (50)-(59) with $\lambda=1$ $(T=1)$.

\begin{tabular}{lcc}
\hline$h$ & $E W(\tau, h)$ & Order \\
\hline $1 / 20$ & $1.1049 e-002$ & - \\
$1 / 30$ & $4.8033 e-003$ & 2.0545 \\
$1 / 40$ & $2.6446 e-003$ & 2.0745 \\
$1 / 50$ & $1.6725 e-003$ & 2.0462 \\
$1 / 60$ & $1.1536 e-003$ & 2.0461 \\
\hline
\end{tabular}

TABLE 4: Numerical convergence of scheme (50)-(59) with $\lambda=2$ $(T=1)$.

\begin{tabular}{lcc}
\hline$h$ & $E W(\tau, h)$ & Order \\
\hline $1 / 30$ & $2.1489 e-002$ & - \\
$1 / 40$ & $1.2564 e-002$ & 1.8656 \\
$1 / 50$ & $7.9166 e-003$ & 2.0699 \\
$1 / 60$ & $5.4036 e-003$ & 2.0946 \\
$1 / 70$ & $3.9183 e-003$ & 2.0850 \\
\hline
\end{tabular}

Theorem 4. For any fixed grid ratio $\lambda=\tau / h$, the difference scheme (50)-(59) is stable with respect to the initial value $\varphi, \psi$, and the source term $f$. More precisely, we have

$$
\left\|\delta_{t} w^{k+(1 / 2)}\right\|^{2}+\frac{1}{2}\left(\left|w^{k+1}\right|_{1}^{2}+\left|w^{k}\right|_{1}^{2}\right) \leq \exp \left(\frac{3}{2} T\right)\left(|\varphi|_{1}^{2}+c\|\psi\|^{2}+\frac{c}{9}\left\|f^{0}\right\|^{2}+\frac{3 \tau}{2} \sum_{l=1}^{k}\left\|f^{l}\right\|^{2}\right), \quad 0 \leq k \leq n-1
$$




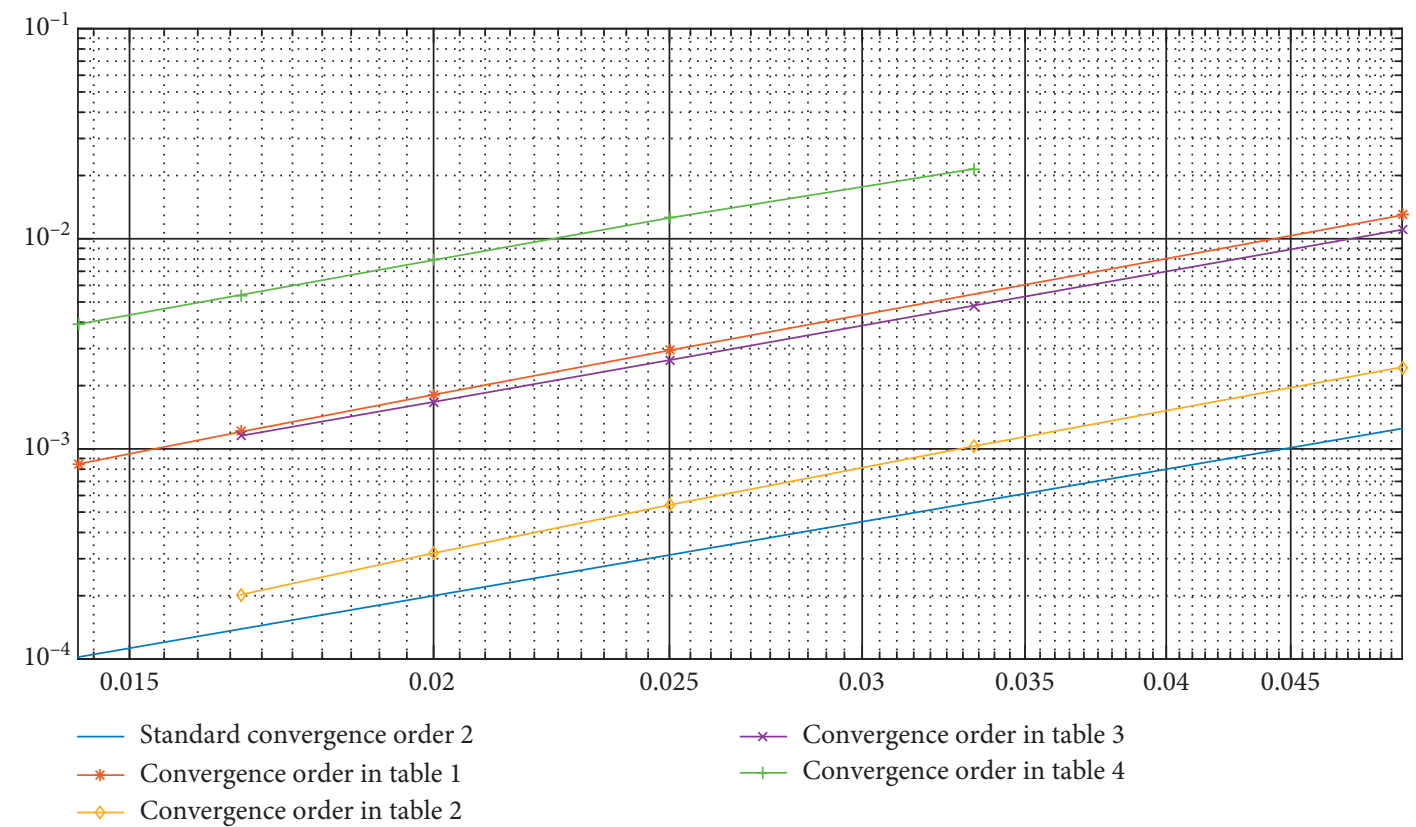

Figure 2: The curve of convergence order in Tables 1-4.

where $c=\max \{2,(1 / \lambda)\}$.

$$
\left\|\delta_{t} w^{k+(1 / 2)}\right\|^{2}+\frac{1}{2}\left(\left|w^{k+1}\right|_{1}^{2}+\left|w^{k}\right|_{1}^{2}\right) \leq \exp \left(\frac{3}{2} T\right)\left(E^{0}+\frac{3 \tau}{2} \sum_{l=1}^{k}\left\|f^{l}\right\|^{2}\right), \quad 0 \leq k \leq n-1,
$$

where

$$
\begin{aligned}
E^{0}= & \left\|\delta_{t} w^{1 / 2}\right\|^{2}+\frac{1}{2}\left(\left|w^{1}\right|_{1}^{2}+\left|w^{0}\right|_{1}^{2}\right) \\
\leq & |\varphi|_{1}^{2}+2 h^{2} \sum_{i, j=1}^{m-1} \psi_{i j}^{2}+\frac{h^{3}}{2 \tau} \sum_{j=1}^{m-1} \psi_{m j}^{2}+\frac{h^{3}}{2 \tau} \sum_{i=1}^{m-1} \psi_{i m}^{2}+\frac{h^{3}}{8 \tau} \psi_{m m}^{2} \\
& +\frac{\tau^{2} h^{2}}{2} \sum_{i, j=1}^{m-1}\left(f_{i j}^{0}\right)^{2}+\frac{\tau h^{3}}{8} \sum_{j=1}^{m-1}\left(f_{m j}^{0}\right)^{2}+\frac{\tau h^{3}}{8} \sum_{i=1}^{m-1}\left(f_{i m}^{0}\right)^{2}+\frac{\tau h^{3}}{32}\left(f_{m m}^{0}\right)^{2} .
\end{aligned}
$$

Taking account into the fact that $\tau \leq(2 / 3)$, $h=(\tau / \lambda) \leq(2 / 3 \lambda)$, there exists constant $c=\max \{2,(1 / \lambda)\}$ such that

$$
E^{0} \leq|\varphi|_{1}^{2}+c\|\psi\|^{2}+\frac{c}{9}\left\|f^{0}\right\|^{2},
$$

which ends the proof.

\section{Numerical Result}

In order to test the convergence order of proposed difference schemes (50)-(59), we consider a two-dimensional damped wave equation, in which the source function $f(x, y, t)$ is defined by
Proof. Suppose that the solution of difference scheme (50)-(59) is $\left\{w_{i j}^{k} \mid 0 \leq i, j \leq m, 0 \leq k \leq n\right\}$, then for any $\lambda$ we can get similarly to Theorem 1

$$
f(x, y, t)=\frac{243 \pi^{2}}{16} \exp \left(-\frac{9}{4} \pi t\right) \sin \left(\frac{9}{4} \pi x\right) \sin \left(\frac{9}{4} \pi y\right),
$$

with initial conditions $\varphi(x, y)$ and $\psi(x, y)$ designed as

$$
\begin{aligned}
& \varphi(x, y)=\sin \left(\frac{9}{4} \pi x\right) \sin \left(\frac{9}{4} \pi y\right), \\
& \psi(x, y)=-\frac{9}{4} \pi \sin \left(\frac{9}{4} \pi x\right) \sin \left(\frac{9}{4} \pi y\right) .
\end{aligned}
$$

For this specified problem, the exact solution is

$$
w(x, y, t)=\exp \left(-\frac{9}{4} \pi t\right) \sin \left(\frac{9}{4} \pi x\right) \sin \left(\frac{9}{4} \pi y\right) .
$$

For the source term and initial conditions defined above, the evolution mesh and contour map of the numerical solution of difference schemes (50)-(59) with $N=40$ and $T=0.5$ are shown in Figure 1.

Let us denote the error by

$$
E W(\tau, h)=\|w(\tau, h)-W(\tau, h)\| .
$$

Table 1 provides the computed results with a fixed $h=1 / 100$. The data demonstrate that the temporal convergence order of difference scheme (50)-(59) is second order in discrete $L^{2}$-norm, which is in accord with 
theoretical analysis of Theorem 3 . When the temporal size is fixed with $\tau=1 / 200$, Table 2 gives the discrete $L^{2}$-errors and numerical convergence orders in space direction. From these data, we can see that the spatial convergence order of difference scheme (50)-(59) is also second order, which is in agreement with theoretical results in Theorem 3.

The convergence results with fixed grid ratio $\lambda=1$ and $\lambda=2$ are shown in Tables 3 and 4, respectively. For these two cases, the proposed difference scheme (50)-(59) can preserve both the convergence and stability. From the numerical results, we can verify the unconditional stability of difference scheme (50)-(59). Also for other different values of $\lambda$, the same stability results can be drawn, which are accordant with Theorem 4 . The curve of convergence order in Tables 1-4 is shown in Figure 2.

\section{Conclusion}

In this paper, a finite difference scheme is constructed for the two-dimensional wave equation with a special boundary condition, damped boundary condition. By introducing a proper discrete $L^{2}$-norm, the proposed finite difference scheme is proved to be second-order convergent in both time and space and unconditionally stable with respect to both initial conditions and source term. The theoretical analysis method can be applied to three-dimensional problems accordingly.

\section{Data Availability}

No data were used to support this study.

\section{Conflicts of Interest}

The authors declare that they have no conflicts of interest.

\section{Acknowledgments}

This work was supported by the NSF of China (nos. 11901365 and 11772177).

\section{References}

[1] J. L. Lions, "Exact controllability, stabilization and perturbations for distributed systems," SIAM Review, vol. 30, no. 1, pp. 1-68, 1988.

[2] H. T. Banks, S. L. Keeling, and R. J. Silcox, "Optimal control techniques for active noise suppression," in Proceedings of the 27th IEEE Conference on Decision and Control, December 1988.

[3] T. Dupont, " $L^{2}$ estimates for galerkin methods for second order hyperbolic equations," SIAM Journal on Numerical Analysis, vol. 10, no. 5, pp. 880-889, 1973.

[4] E. H. Georgoulis, O. Lakkis, and C. Makridakis, "A posteriori $L\left(L^{2}\right)$-error bounds for finite element approximations to the wave equation," IMA Journal of Numerical Analysis, vol. 33, no. 4, pp. 1245-1264, 2013.

[5] R. Eymard, T. Gallouët, M. Ghilani, and R. Herbin, "Error estimates for the approximate solutions of a nonlinear hyperbolic equation given by finite volume schemes," IMA
Journal of Numerical Analysis, vol. 18, no. 4, pp. 563-594, 1998.

[6] A. Bradji, "A theoretical analysis of a new finite volume scheme for second order hyperbolic equations on general nonconforming multidimensional spatial meshes," Numerical Methods for Partial Differential Equations, vol. 29, no. 1, pp. 1-39, 2013.

[7] R. M. Beam and R. F. Warming, "An implicit finite-difference algorithm for hyperbolic systems in conservation-law form," Journal of Computational Physics, vol. 22, no. 1, pp. 87-110, 1976.

[8] R. K. Mohanty, "An unconditionally stable difference scheme for the one-space-dimensional linear hyperbolic equation," Applied Mathematics Letters, vol. 17, no. 1, pp. 101-105, 2004.

[9] M. J. Grote, A. Schneebeli, and D. Schötzau, "Discontinuous galerkin finite element method for the wave equation," SIAM Journal on Numerical Analysis, vol. 44, no. 6, pp. 2408-2431, 2006.

[10] D. Appelö and T. Hagstrom, "A new discontinuous galerkin formulation for wave equations in second-order form," SIAM Journal on Numerical Analysis, vol. 53, no. 6, pp. 2705-2726, 2015.

[11] B. Cockburn and V. Quenneville-Bélair, "Uniform-in-time superconvergence of the HDG methods for the acoustic wave equation," Mathematics of Computation, vol. 83, no. 285, pp. 65-85, 2014.

[12] L. C. Cowsat, T. F. Dupont, and M. F. Wheeler, "A priori estimates for mixed finite element methods for the wave equation," Computer Methods in Applied Mechanics and Engineering, vol. 82, no. 1-3, pp. 205-222, 1990.

[13] C. W. Shu, Essentially Non-oscillatory and Weighted Essentially Non-oscillatory Schemes for Hyperbolic Conservation Laws, Springer, Berlin, Germany, 1998.

[14] L. Ivan, H. De Sterck, A. Susanto, and C. P. T. Groth, "Highorder central eno finite-volume scheme for hyperbolic conservation laws on three-dimensional cubed-sphere grids," Journal of Computational Physics, vol. 282, pp. 157-182, 2015.

[15] J. Zhu and J. Qiu, "A new fifth order finite difference weno scheme for solving hyperbolic conservation laws," Journal of Computational Physics, vol. 318, pp. 110-121, 2016.

[16] F. Zhao, L. Pan, Z. Li, and S. Wang, "A new class of high-order weighted essentially non-oscillatory schemes for hyperbolic conservation laws," Computers \& Fluids, vol. 159, pp. 81-94, 2017.

[17] J. Zhu and C.-W. Shu, "A new type of multi-resolution weno schemes with increasingly higher order of accuracy on triangular meshes," Journal of Computational Physics, vol. 392, pp. 19-33, 2019.

[18] A. R. Appadu, M. Z. Dauhoo, and S. D. D. V. Rughooputh, "Control of numerical effects of dispersion and dissipation in numerical schemes for efficient shock-capturing through an optimal Courant number," Computers \& Fluids, vol. 37, no. 6, pp. 767-783, 2008.

[19] A. R. Appadu, "Some applications of the concept of minimized integrated exponential error for low dispersion and low dissipation," International Journal for Numerical Methods in Fluids, vol. 68, no. 2, pp. 244-268, 2012.

[20] A. R. Appadu, "Optimised composite numerical schemes in 2D for hyperbolic conservation laws," International Journal for Numerical Methods in Fluids, vol. 69, no. 9, pp. 1522-1549, 2012.

[21] A. R. Appadu and A. A. I. Peer, "Optimized weighted essentially nonoscillatory third-order schemes for hyperbolic conservation laws," Journal of Applied Mathematics, vol. 2013, pp. 1-12, 2013. 
[22] A. R. Appadu and S. N. N. Nguetchue, "The technique of MIEELDLD as a measure of the shock-capturing property of numerical methods for hyperbolic conservation laws," Progress in Computational Fluid Dynamics-An International Journal, vol. 15, no. 4, pp. 247-264, 2015.

[23] L. Kong, P. Zhu, Y. Wang, and Z. Zeng, "Efficient and accurate numerical methods for the multidimensional convectiondiffusion equations," Mathematics and Computers in Simulation, vol. 162, pp. 179-194, 2019.

[24] D. Deng and C. Zhang, "Application of a fourth-order compact ADI method to solve a two-dimensional linear hyperbolic equation," International Journal of Computer Mathematics, vol. 90, no. 2, pp. 273-291, 2013.

[25] H.-L. Liao and Z.-Z. Sun, "A two-level compact ADI method for solving second-order wave equations," International Journal of Computer Mathematics, vol. 90, no. 7, pp. 14711488, 2013.

[26] A. K. Pani and J. Y. Yuan, "Mixed finite element method for a strongly damped wave equation," Numerical Methods for Partial Differential Equations, vol. 17, no. 2, pp. 105-119, 2001.

[27] S. Karaa, "Error estimates for finite element approximations of a viscous wave equation," Numerical Functional Analysis and Optimization, vol. 32, no. 7, pp. 750-767, 2011.

[28] L. Gao, D. Liang, and B. Zhang, "Error estimates for mixed finite element approximations of the viscoelasticity wave equation," Mathematical Methods in the Applied Sciences, vol. 27, no. 17, pp. 1997-2016, 2004.

[29] R. K. Sinha, A. K. Pani, and S. K. Chung, "The effect of spatial quadrature on the semidiscrete finite element galerkin method for a strongly damped wave equation," Numerical Functional Analysis and Optimization, vol. 24, no. 3-4, pp. 311-325, 2003.

[30] L. C. Cowsar, T. F. Dupont, and M. F. Wheeler, "A priori estimates for mixed finite element approximations of secondorder hyperbolic equations with absorbing boundary conditions," SIAM Journal on Numerical Analysis, vol. 33, no. 2, pp. 492-504, 1996.

[31] S. Ş. Şener, Y. Saraç, and M. Subaşı, "Weak solutions to hyperbolic problems with inhomogeneous dirichlet and neumann boundary conditions," Applied Mathematical Modelling, vol. 37, no. 5, pp. 2623-2629, 2013.

[32] Z. Z. Sun, Numerical Methods for Partial Differential Equations, Science Press, China, 2012, in Chinese. 